\title{
miR-148b-3p inhibits malignant biological behaviors of human glioma cells induced by high HOTAIR expression
}

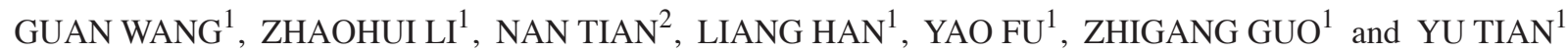 \\ ${ }^{1}$ Department of Neurosurgery, China-Japan Union Hospital of Jilin University, Changchun, Jilin 130033; \\ ${ }^{2}$ Department of Cell Biology, College of Life Science, Zhejiang Chinese Medical University, \\ Hangzhou, Zhejiang 310053, P.R. China
}

Received December 4, 2015; Accepted May 26, 2016

DOI: $10.3892 / \mathrm{ol} .2016 .4743$

\begin{abstract}
Increasing evidence suggests that long non coding (lnc)RNA and microRNA (miRNA/miR) both regulate the expression of key genes in tumorigenesis and have considerable theranostic potential. Rapid advances in bioinformatics indicate that miRNA may potentially interact with lncRNA to modulate their regulatory roles. miR-148b-3p has been reported to have a vital role in regulating tumor progression. However, the expression pattern of miR-148b-3p in glioma remains largely unknown, and interactions between miR-148b-3p and lncRNA has yet to be identified. The aim of the present study was to insight into the regulatory role of miR-148b-3p in glioma. Using online software, the HOTAIR gene was identified as a possible IncRNA target of miR-148b-3p in the present study. siRNA was used to suppress the expression of HOTAIR and reverse transcription-quantitative polymerase chain reaction was used to detect the expression of miR-148b-3p. The results confirmed that HOTAIR mRNA expression was inversely correlated with miR-148b-3p expression in A172 glioma cells. Furthermore, a 3-(4,5-dimethylthiazol-2-yl)-2,5-diphenyltetrazolium bromide assay was performed to detect the viability of cells, flow cytometry was performed to test cell cycle and a matrigel invasion assay was performed to test cell invasion. The results showed that HOTAIR promotes factors associated with malignancy, including cell proliferation, cell cycle progression and invasion, whereas miR-148b-3p suppresses malignancy. Bioinformatics and luciferase reporter assays showed that miR-148b-3p modulates HOTAIR expression by directly targeting the HOTAIR gene sequence. In summary, the results indicated
\end{abstract}

Correspondence to: Dr Yu Tian, Department of Neurosurgery, China-Japan Union Hospital of Jilin University, 126 Xiantai Street, Changchun, Jilin 130033, P.R. China

E-mail: tianyu@jlu.edu.cn

Abbreviations: CNS, central nervous system; DMEM, Dulbecco's modified Eagle medium; MTT, 3-(4,5-dimethylthiazol-2-yl)-2,5-diphenyltetrazolium bromide

Key words: long non-coding RNA/microRNA interaction, HOTAIR, miR-148b-3p, Matrigel, glioblastoma that miR-148b-3p inhibits malignant biological behaviors of glioma cells by directly targeting HOTAIR. The current data provide important evidence for understanding the key roles of the lncRNA miRNA functional network in glioma.

\section{Introduction}

Advances in contemporary molecular biology have revealed that a large number of RNAs that have no protein-coding ability [non-coding (nc)RNAs] exist in various organs and tissues (1-4). Many of these ncRNAs are key factors in gene regulation and are important for normal cellular function as well as disease pathogenesis (5). Among the different classes of ncRNAs, long ncRNAs (lncRNAs), which are most commonly defined as non-protein-coding RNA molecules of $>200$ nucleotides, have recently received increasing attention. IncRNAs can be transcribed from intergenic, intronic and imprinted loci, or from overlapping or antisense loci adjacent to protein-coding genes. These diverse transcription patterns of lncRNAs have significant implications for their various functions, including imprinting, DNA methylation and X-chromosome dosage compensation, as well as transcriptional, post-transcriptional and epigenetic regulation (6-11). The majority of lncRNAs are transcribed in a developmentally-regulated and cell type-specific manner $(12,13)$, particularly in the central nervous system (CNS), where over half of all lncRNAs are expressed (13-15). Therefore, IncRNAs are now considered to be highly important for mediating CNS form and function, and alteration of their expression may cause certain CNS pathologies, such as Alzheimer's disease, multiple sclerosis, Down's syndrome, schizophrenia and brain tumors (16).

Gliomas are the most common and aggressive type of primary adult brain tumor, with an prevalence of 3-6 per 100,000 population in China (17). Their highly malignant and invasive nature gives rise to a median survival time of $<15$ months for patients with glioblastoma (GBM) undergoing conventional treatment. The pathogenesis of glioma is complex and involves the aberrant activation of proto-oncogenes and the inactivation of tumor suppressor genes (18-20). Growing evidence in the literature has revealed that aberrant expression of lncRNAs in glioma influence cell proliferation, apoptosis and invasion through interactions with different molecules and diverse signaling pathways (21-23), thereby acting as critical 
components in the progression of gliomas. For example, H19, a well-characterized lncRNA in glioma, drives tumor transformation and contributes to malignant glioma phenotypes through binding with transcription factor c-Myc $(22,24)$. $C R N D E$, the most upregulated lncRNA in GBM (16), regulates gene expression through histone methylation/demethylation epigenetic changes; these histone modifications are induced via interaction with chromatin modifying complexes CoREST and polycomb repressive complex 2 (PRC2) (25). In addition, $M E G 3$, a tumor suppressor lncRNA $(23,26)$, appears to have an anti-proliferative function in glioma through the suppression of MDM2 and subsequent activation of the p53 signaling pathway (27). HOTAIR, which also participates in epigenetic regulation through PRC2, was demonstrated to be closely associated with tumor staging, poor prognosis and the molecular subtype of glioma (17), with knockdown of HOTAIR in glioma cells exerting a tumor suppressive function accompanied by significant downregulation of cell-cycle related genes $(28,29)$. Despite the data obtained from the previous aforementioned studies, the exact mechanisms by which lncRNAs regulate the development of gliomas remain largely unclear.

Several studies have suggested that the interaction between lncRNAs and microRNAs (miRNA/miRs) may have a regulatory role in cancer (30-33). For example, MEG3 is methylation-dependent tissue-specific IncRNA that is regulated by miR-29a and has been reported to contribute to hepatocellular carcinoma growth (34). Furthermore, UCA1, an oncogene lncRNA in breast cancer, modulates breast cancer cell growth and apoptosis by decreasing tumor suppressive miR-143 (35), and miR-148b-3p, a member of the miR-148/152 family, was underexpressed (reduced compared with normal cells) in several cancer cell lines (36-38). In a study by Zhang et al, miR-148b-3p was found to induce cell apoptosis by activating caspase- 3 and caspase- 9 , inducing $S$ phase arrest by regulating cyclin D1 and p21, and inhibiting cell invasion (39). Although a number of mRNA targets of miR-148b-3p have been identified, interaction between miR-148b-3p and an lncRNA has not been reported to date. Using the online software program starBase v2.0, the present study identified HOTAIR as a potential lncRNA target of miR-148b-3p. Subsequently, the current study showed that mutated HOTAIR promotes the aggressiveness of A172 glioma cells, and its was determined that miR-148b-3p binds HOTAIR in a sequence-specific manner. Furthermore, miR-148b-3p reduced proliferation, cell cycle progression and invasion of A172 cells through the suppression of HOTAIR expression. Thus, the current data, at least in part, contributes insight into the development of glioma.

\section{Materials and methods}

Human tissue samples and cell lines. mRNA and miRNA expression microarray data from 180 samples were downloaded from the Gene Expression Omnibus website (http://www.ncbi.nlm.nih.gov/geo/; accession no. GSE4290). The data were compiled from 23 non-tumor, 26 astrocytoma (7 grade II, 19 grade III), 50 oligodendroglioma (38 grade II, 12 grade III) and 81 GBM samples. The tumor sample expression profile, including HOTAIR expression data, was also downloaded. HA1800 human astrocytes and A172 glioma cells were purchased from the Cell Resource Center of
Shanghai Institute of Life Sciences (Shanghai, China). The cell lines were cultured in Dulbecco's modified Eagle's medium (DMEM; Gibco; Thermo Fisher Scientific, Inc., Waltham, MA, USA) supplemented with $10 \%$ fetal calf serum (Gibco; Thermo Fisher Scientific, Inc.), penicillin (100 U/ml) and streptomycin $(100 \mathrm{mg} / \mathrm{ml})$ at $37^{\circ} \mathrm{C}$ in $5 \% \mathrm{CO}_{2}$.

Reverse transcription-quantitative polymerase chain reaction (RT-qPCR). Total RNA was extracted from the cells using TRIzol Reagent (Invitrogen; Thermo Fisher Scientific, Inc.), according to the manufacturer's instructions. Briefly, $1 \mathrm{ml}$ TRIzol per $5 \times 10^{5}$ cells was added to cells, prior to adding $0.2 \mathrm{ml}$ of chloroform per $1 \mathrm{ml}$ TRIzol. The mixture was mixed vigorously by hand and allowed to stand for 2-3 min at room temperature. The mixture was then centrifuged at $10,000 \mathrm{x} g$ for $10 \mathrm{~min}$ at $4^{\circ} \mathrm{C}$. The upper clear phase was transferred to a fresh tube and $0.5 \mathrm{ml}$ isopropanol per $1 \mathrm{ml}$ of the clear phase was added, which was mixed vigorously by rapid shaking and left to stand for $10 \mathrm{~min}$. The precipitated RNA was collected by centrifugation at $10,000 \mathrm{x} \mathrm{g}$ for $10 \mathrm{~min}$ at $4^{\circ} \mathrm{C}$ and then carefully decanting/pipetting the supernatant. The RNA precipitate was washed once with $70 \%$ ethanol, dissolved in $25 \mu$ l RNase free water, and then stored at $-80^{\circ} \mathrm{C}$. The concentration of the Recombinant DNase I RNase-free (Takara Biotechnology Co., Ltd., Dalian, China) used to treat the RNA sample was 5 units $/ \mu 1$.

cDNA was synthesized using the HiFi-MMLV cDNA kit (Beijing ComWin Biotech Co., Ltd., Beijing, China) and qPCR was conducted using the UltraSYBR Mixture (Beijing ComWin Biotech Co., Ltd.). Briefly, $5 \mu \mathrm{g}$ purified RNA sample was mixed with Primer Mix, dNTP Mix, DTT, RT-buffer, HiFi-MMLV and RNase-free water using a pulled pipette (total volume, $20 \mu \mathrm{l}$ ). All qRT-PCR reactions were run in a StepOnePlus $^{\text {TM }}$ Real-Time PCR System (Applied Biosystems; Thermo Fisher Scientific, Inc.). The mixture was then incubated at $42^{\circ} \mathrm{C}$ for $30-50 \mathrm{~min}$ and then $85^{\circ} \mathrm{C}$ for $5 \mathrm{~min}$. The RT products were quickly centrifuged and stored at $-20^{\circ} \mathrm{C}$. No cDNA was used as a negative control. To amplify hsa-miR-148b-3p cDNA, specific RT primers were used based on its sequence, and the U6 RT primer for was the same as the U6 reverse PCR primer. The hsa-miR-148b-3p RT primer was 5'-GTTGGCTCTGGT GCAGGGTCCGAGGTATTCGCACCAGAGCCAACACAA AG-3'. PCR primers were as follows: Forward, 5'-GGCACC ACACCTTCTACAAT-3' and reverse, 5'-GTGGTGGTGAAG CTGTAGCC-3' for the $\beta$-actin gene; forward, 5'-CAGTGG GGAACTCTGACTCG-3' and reverse, 5'-GTGCCTGGTGCT CTCTTACC-3' for the HOTAIR gene; forward, 5'-CGGTCA GTGCATCACAGAA-3' and reverse, 5'-GTGCAGGGTCCG AGGT-3' for hsa-miR-148b-3p; and forward, 5'-CTCGCT TCGGCAGCACA-3' and reverse, 5'-AACGCTTCACGAATT TGCGT-3' for U6. All primers were synthesized by GenScript (Nanjing, China). The relative fold change in mRNA expression level was calculated using the $2^{-\Delta \Delta \mathrm{Cq}}$ method (40).

Cell transfection. siRNA HOTAIR (siHOTAIR), siRNA negative control ( $\mathrm{siNC}$ ), miR-148b-3p mimic and miR-148b-3p inhibitor were synthesized by Biomics Biotechnologies Co., Ltd. (Nantong, China). The sequences were as follows: Sense, 5'-CCACAUGAACGCCCAGAGAUU-3' and antisense, 5'-AAUCUCUGGGCGUUCAUGUGG-3' for si-HOTAIR (41); and sense, 5'-UUCUCCGAACGUGUCACGUTT-3' and 
A

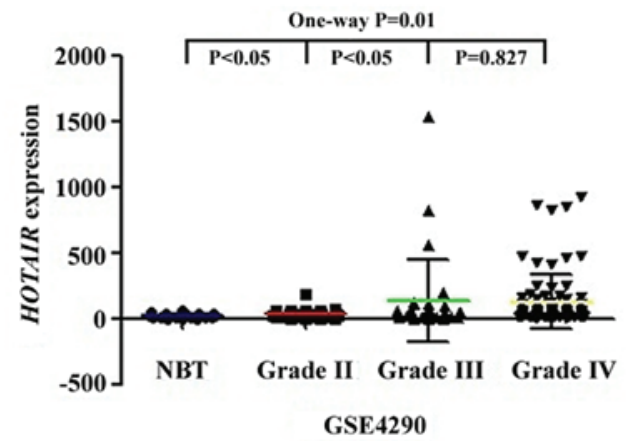

B

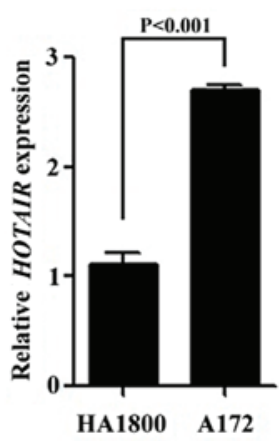

Figure 1. HOTAIR expression in glioma tissues and cells. (A) Expression levels of HOTAIR in glioma tissues from an NCBI GEO dataset, as analyzed by whole genome gene expression profiling. (B) mRNA expression level of HOTAIR in HA1800 and A172 cell lines, as determined by reverse transcription-quantitative polymerase chain reaction. Data are presented as the mean \pm standard deviation of three independent experiments. NBT, normal brain tissue.

antisense, 5'-ACGUGACACGUUCGGAGA ATT-3' for si-NC (22). Cells were grown tuntil they reached $10^{5}$ in number in 6-well plates prior to transfection. One day before transfection, the control group cells were plated at the same density as the si-HOTAIR and si-NC groups. The cells were transfected using Lipofectamine 2000 (Invitrogen; Thermo Fisher Scientific, Inc.), according to the manufacturer's instructions.

Cell viability assay. The effect of HOTAIR downregulation on the viability of A172 cells was assessed using the 3-(4,5-dimethylthiazol-2-yl)-2,5-diphenyltetrazolium bromide (MTT) assay. Briefly, cells were trypsinized and seeded at a density of $1 \times 10^{4}$ cells/well in 96-well plates immediately after siRNA transfection. Then, $10 \mu \mathrm{l}$ MTT solution $(5 \mathrm{mg} / \mathrm{ml})$ was added and the plates were incubated for an additional $4 \mathrm{~h}$ at $37^{\circ} \mathrm{C}$. Following removal of the medium, formazan crystals were dissolved in $150 \mu \mathrm{l}$ dimethyl sulfoxide. The absorbance of the MTT formazan was measured at $550 \mathrm{~nm}$ using a SpectraMax M3 microplate reader (Molecular Devices, LLC, Sunnyvale, CA, USA). Experiments were repeated three times using 6 wells for each treatment to ensure the reproducibility of results.

Flow cytometry analysis (FCM). Cells were separately harvested $0,24,48$ and $72 \mathrm{~h}$ after siRNA transfection, fixed in $70 \%$ ethanol, and stained with propidium iodide (Nanjing KeyGen Biotech Co. Ltd., Nanjing, China) containing RNase A (1 mg/ml; Takara Biotechnology Co., Ltd.) for $30 \mathrm{~min}$ at $37^{\circ} \mathrm{C}$. Subsequently, $500 \mu \mathrm{l}$ of cells was filtered through $200-\mu \mathrm{m}$ mesh sieves, and the cell cycle profiles were assayed using a Guava easyCyte 8 Flow Cytometer (EMD Millipore, Billerica, MA, USA).

Matrigel invasion assay. Transwell inserts (diameter, $6.5 \mathrm{~mm}$ ) with a pore size of $8 \mu \mathrm{M}$ (Corning Incorporated, Corning, NY, USA) were coated with Matrigel (100 $\mu \mathrm{g} / \mathrm{well}$; BD Biosciences, San Jose, CA, USA) and placed into the wells of 24-well culture plates. Following transfection with siRNA for $48 \mathrm{~h}, 1 \times 10^{4}$ cells were transferred into the top of the invasion chambers in serum-free DMEM, and DMEM containing 20\% fetal calf serum was added to the lower chambers. After $24 \mathrm{~h}$ of incubation at $37^{\circ} \mathrm{C}$, non-invasive cells were removed with a cotton swab, and the invading cells were fixed with $4 \%$ paraformaldehyde for $15 \mathrm{~min}$, stained with Giemsa for $20 \mathrm{~min}$ at room temperature and observed under an ECLIPSE Ti S microscope (Nikon, Tokyo, Japan). Experiments were independently repeated three times.

Plasmid construction. To verify whether miR-148b-3p regulates HOTAIR as a direct target, a predicted binding site (12 bp) for miR-148b-3p was identified using the online software program starBase v2.0 (42). The human HOTAIR fragment containing the putative binding sites for miR-148b-3p was synthesized, annealed and inserted into the NotI and XbaI restriction sites of the pmirGLO luciferase reporter vector (Promega, Madison, WI, USA), downstream of the luciferase gene, to generate the recombinant vectors pmirGLO-wild-type (WT) and pmirGLO-mutant (MUT). For the pmirGLO-MUT construct, 12 mismatches were introduced into the HOTAIR sequence, producing a change of GATGCATTTTCTGTG CACTGG to GATGCACCTCCCACATGTCAG; therefore, a human HOTAIR fragment containing mutated binding sites was synthesized. The constructs were validated by Sanger sequencing by Sangon Biotech Co., Ltd. (Shanghai, China).

Luciferase reporter assay. For the luciferase reporter assay, A172 cells were co-transfected with miRNA (miR-148b-3p mimics or miR-148-3p mimic negative control; Biomics Biotechnologies Co., Ltd., Nantong, China) and reporter vectors (pmirGLO-WT or pmirGLO-MUT) using Lipofectamine 2000. Luciferase activity was assayed $48 \mathrm{~h}$ after transfection using a Dual-Luciferase Reporter Assay system (Beyotime Institute of Biotechnology, Haimen, China). The values were normalized to those obtained for miRNA negative control transfection. All transfection experiments were performed in triplicate.

Statistical analysis. All statistical analyses were performed using SPSS software version 18.0 (SPSS, Inc., Chicago, IL, USA). Data are expressed as means \pm standard deviation of experiments performed in triplicate. HOTAIR expression in normal tissues and tumor tissues was compared using analysis of variance (ANOVA) followed by Student-Newman-Keuls analysis. GraphPad Prism version 5.0 (GraphPad Software, La Jolla, CA, USA) was used for the graphing. Statistical analysis on other experiments was performed using one-way ANOVA and the Student-Newman-Keuls test for multiple comparisons, 
A

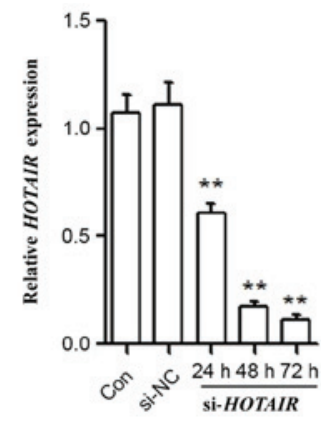

B

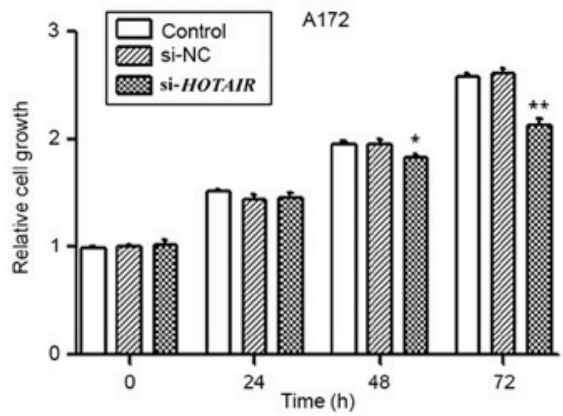

C
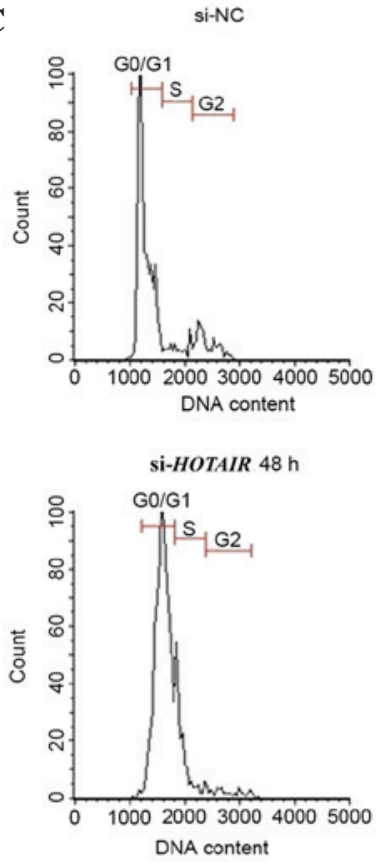

si-HOTAIR $24 \mathrm{~h}$
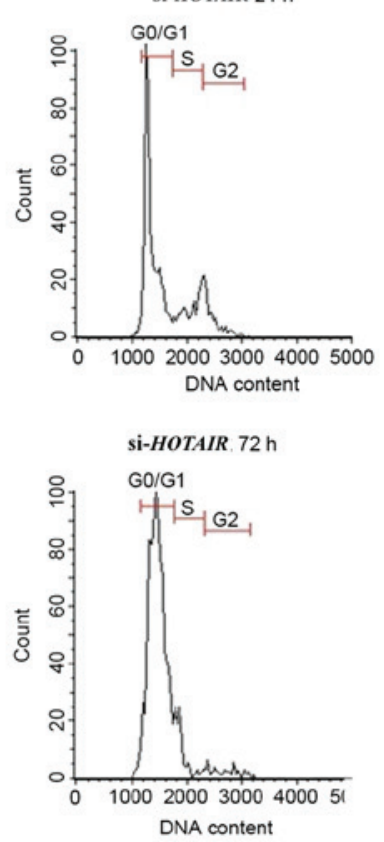

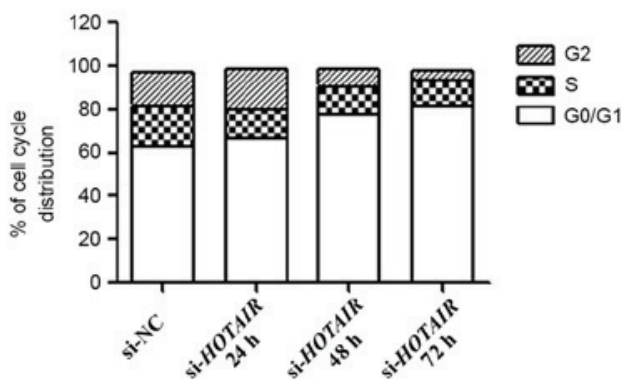

D

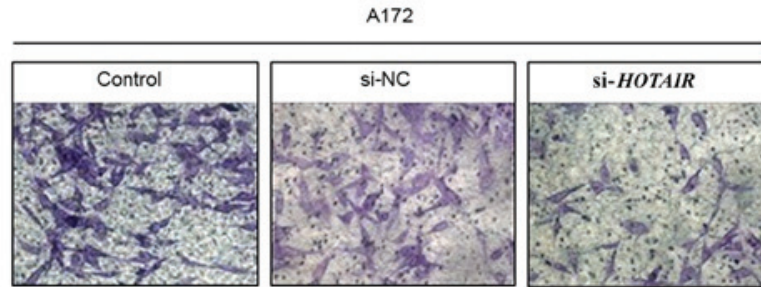

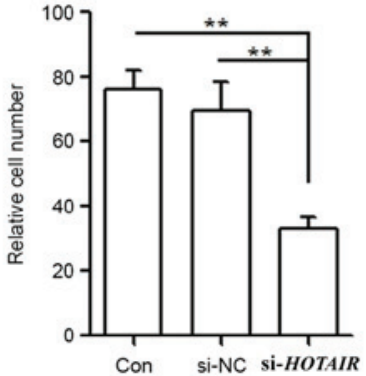

Figure 2. Silencing HOTAIR inhibits the malignant properties of A172 cells. (A) Knockdown of the HOTAIR gene in A172 cells by siRNA transfection. Relative gene expression was determined using the $\Delta \Delta \mathrm{Cq}$ method $\left(2^{-\Delta \Delta \mathrm{Cq}}\right)$. ${ }^{* *} \mathrm{P}<0.001$. (B) Effect of HOTAIR knockdown on A172 cell growth. Cell proliferation was detected at the indicated time points following transfection using the MTT assays. (C) Effect of HOTAIR knockdown on the A172 cell cycle. Cell cycle distribution was measured by propidium iodide staining followed by flow cytometry. (D) Effect of HOTAIR knockdown on A172 cell invasion. The representative microscopic images (Giemsa staining; magnification, $\mathrm{x} 400$ ) and quantification of invaded cells were analyzed using a Matrigel invasion assay. "P<0.05, ${ }^{* *} \mathrm{P}<0.01$, siHOTAIR groups compared with siNC and untransfected cell groups. Data are presented as the mean \pm standard deviation of three independent experiments. miR, microRNA; si-NC, siRNA-negative control; si-HOTAIR, siRNA HOTAIR.

and unpaired Student's $t$-tests for comparisons between groups. $\mathrm{P}<0.05$ was considered to indicate a statistically significant difference.

\section{Results}

HOTAIR expression in glioma tissues and cells. First, the expression pattern of HOTAIR was analyzed through whole genome gene expression profiling of 157 glioma and 23 normal tissue samples from NCBI GEO data (accession no. GSE4290).
As shown in Fig. 1A, HOTAIR expression was significantly higher in carcinoma tissues compared with in normal tissues $(\mathrm{P}=0.010)$, and grade IV and III tumor tissues both demonstrated a significant increase in HOTAIR transcription levels compared with grade II tumor tissues $(\mathrm{P}=0.047)$. However, no significant difference in HOTAIR expression was observed between grade IV and III samples. Next, HOTAIR expression was detected in HA1800 human astrocytes and A172 glioma cells by RT-qPCR. The HOTAIR mRNA level was significantly increased in A172 cells compared with HA1800 cells 
A

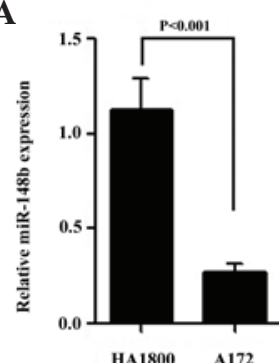

D

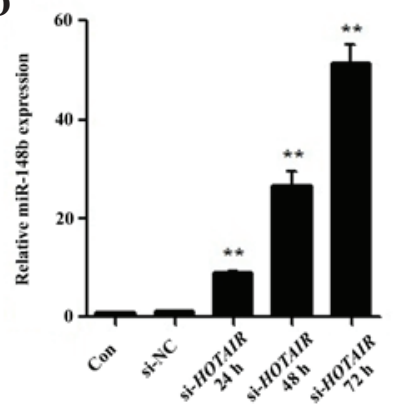

B

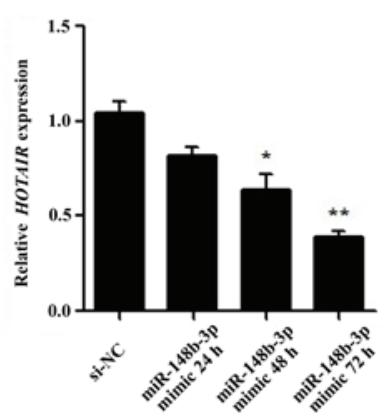

E

$\begin{array}{lcc}\text { HOTAIR } & 5^{\prime} \text {...GATGCATTTTCTG - TGCACTGG...3' } \\ \text { Hsa-miR-148b } & 3^{\prime} \quad \text { TGTTTCAAGACACTACGTGACT } 5^{\prime} \\ \text { HOTAIR } \text {-mutant } & 5^{\prime} \text {...GATGCACCTCCCACATGTCAG...3' }\end{array}$
C

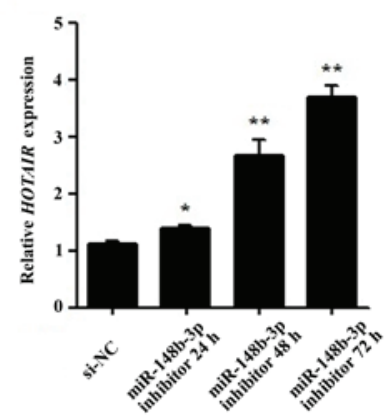

F

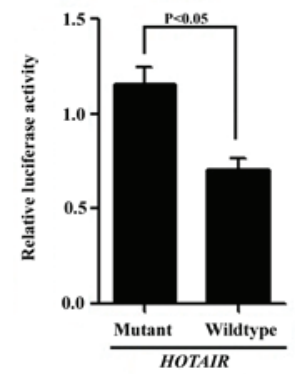

Figure 3. Reciprocal negative regulation of miR-148b-3p and HOTAIR. (A) miR-148b-3p expression in HA1800 and A172 cell lines. (B and C) Effect of miR-148b-3p on HOTAIR expression. A172 cells were transfected with si-NC, (B) miR-148b-3p mimic or (C) miR-148b-3p inhibitor. (D) Upregulation of miR-148b-3p induced by ectopic expression of HOTAIR. A172 cells were transfected with si-NC or si-HOTAIR. At 24, 48 and $72 \mathrm{~h}$ post-transfection, mRNA expression was analyzed by reverse transcription-quantitative polymerase chain reaction. (E) miR-148b-3p binding sequence in HOTAIR. The red letters in the HOTAIR sequence show mutations. (F) A172 cells were co-transfected with the pmirGLO luciferase construct (pmirGLO-mutant or pmirGLO-wild-type) and miR-148b-3p mimic. After $24 \mathrm{~h}$ of incubation, cells were harvested and assayed using a luciferase reporter assay system. ${ }^{*} \mathrm{P}<0.05,{ }^{* *} \mathrm{P}<0.01 \mathrm{vs}$. si-NC. Data are presented as the mean \pm standard deviation of three independent experiments. miR, microRNA. si-NC; siRNA-negative control; si-HOTAIR, siRNA HOTAIR.

$(\mathrm{P}<0.001$; Fig. 1B). These findings indicate that HOTAIR may have an important role in glioma progression.

Silencing HOTAIR inhibits the malignant properties of A172 cells. To further investigate the oncogenic role of HOTAIR in glioma pathogenesis, A172 cell lines were transfected with si-HOTAIR. Successful transfection was confirmed by RT-qPCR (Fig. 2A). Cells were separately collected at $0,24,48$ and $72 \mathrm{~h}$ post-transfection, and cell growth ability was determined using the MTT assay. Silencing of HOTAIR expression significantly reduced growth rates compared with the si-NC and untransfected cell groups at $48(\mathrm{P}=0.012)$ and $72 \mathrm{~h}(\mathrm{P}<0.001)$ post-transfection (Fig. 2B). Additionally, FCM was performed following transfection to assess cell cycle distribution. The results showed that the cell population in the G1 phase was increased but the $\mathrm{S}$ phase population was decreased after HOTAIR gene silencing compared with the results observed for the si-NC cells (Fig. 2C), further indicating that knockdown of HOTAIR expression may suppress cancer cell proliferation. In addition, the effect of HOTAIR knockdown on A172 cell invasion was investigated using a Matrigel invasion assay. The in vitro Matrigel invasion assay revealed that the invasiveness of A172 cells transfected with si-HOTAIR was significantly suppressed compared with the control and si-NC cells ( $\mathrm{P}=0.007$; Fig. 2D).

Reciprocal negative regulation of $m i R-148 b-3 p$ and HOTAIR. RT-qPCR revealed that the expression level of miR-148b-3p was significantly lower in A172 cells compared with HA1800 cells $(\mathrm{P}<0.001$; Fig. 3A). To determine whether miR-148b-3p is able to suppress HOTAIR expression, miR-148b-3p mimic or inhibitor were transfected into A172 cells. As shown in Fig. 3B, the miR-148b-3p mimic reduced HOTAIR expression in a dose-dependent manner, decreasing it by $\sim 62 \% 72 \mathrm{~h}$ after transfection $(\mathrm{P}<0.001)$. By contrast, the miR-148b-3p inhibitor increased the level of HOTAIR in a dose-dependent manner (Fig. 3C). Furthermore, si-HOTAIR transfection significantly increased the expression of miR-148b-3p in A172 cells at all time points $(\mathrm{P}<0.001$; Fig. 3D), indicating that there is a strong inverse correlation between HOTAIR and miR-148b-3p expression levels.

To verify whether miR-148b-3p regulates HOTAIR as a direct target, a predicted binding site for miR-148b-3p was identified using the online software program starBase v2.0 (42) (Fig. 3E) an wild-type or mutant miR-148b-3p target binding sequences were cloned into the pmirGLO luciferase vector. Following co-transfection with the miR-148b-3p mimic in A172 cells, a Dual-Luciferase Assay was performed determine the luciferase activity. The data shows that cells co-transfected with the constructs containing the pmirGLO-WT and miR-148b-3p mimic had significantly lower luciferase activity compared with that of those transfected with pmirGLO-MUT and miR-148b-3p mimic ( $\mathrm{P}=0.007$; Fig. 3F). All the results indicate that miR-148b-3p suppresses HOTAIR by binding to HOTAIR in a sequence-specific manner.

miR-148b-3p inhibits HOTAIR function. To detect whether the aggressiveness of A172 cells could be restored by miR-148b-3p, an MTT assay, FCM and a Transwell invasion assay were performed following transfection with the miR-148b-3p mimic. 
A

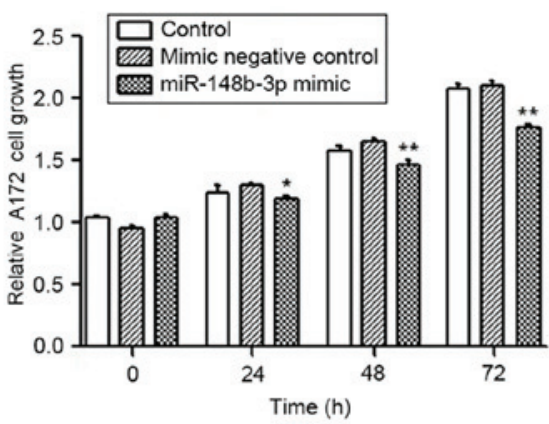

B
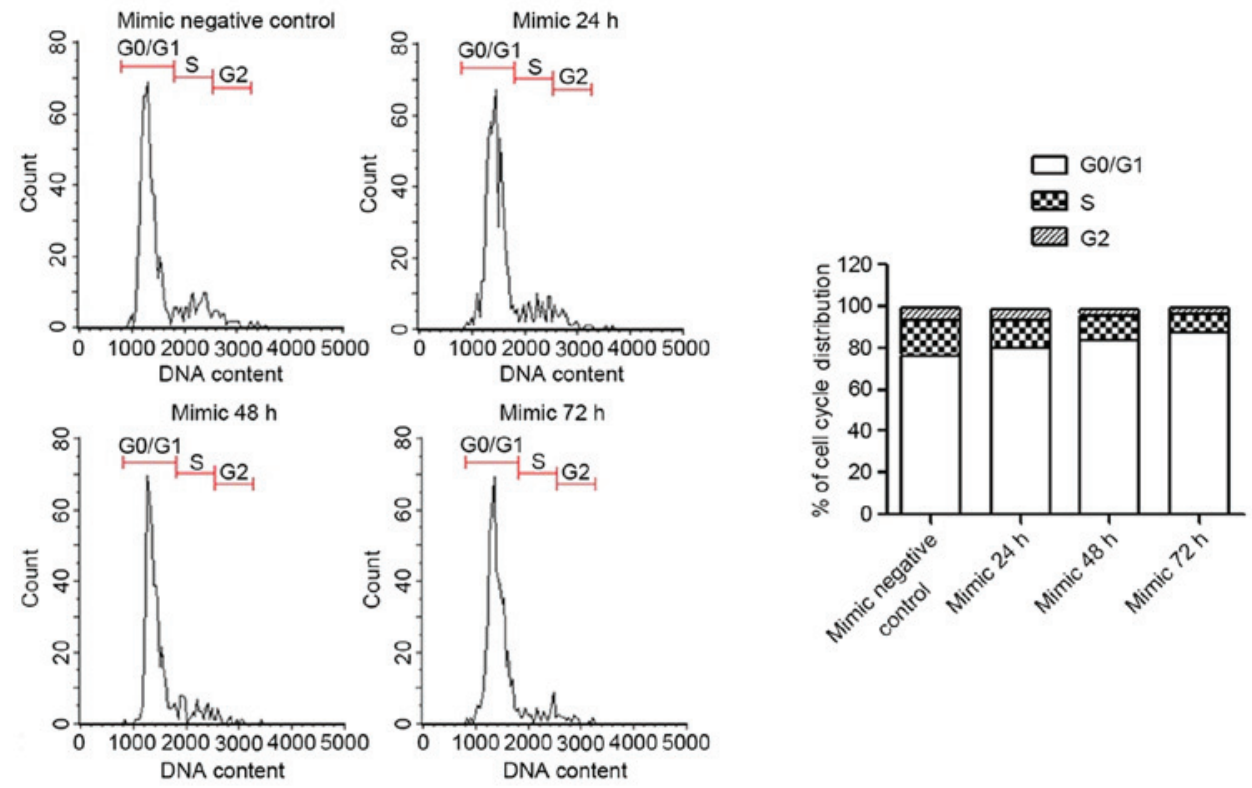

C
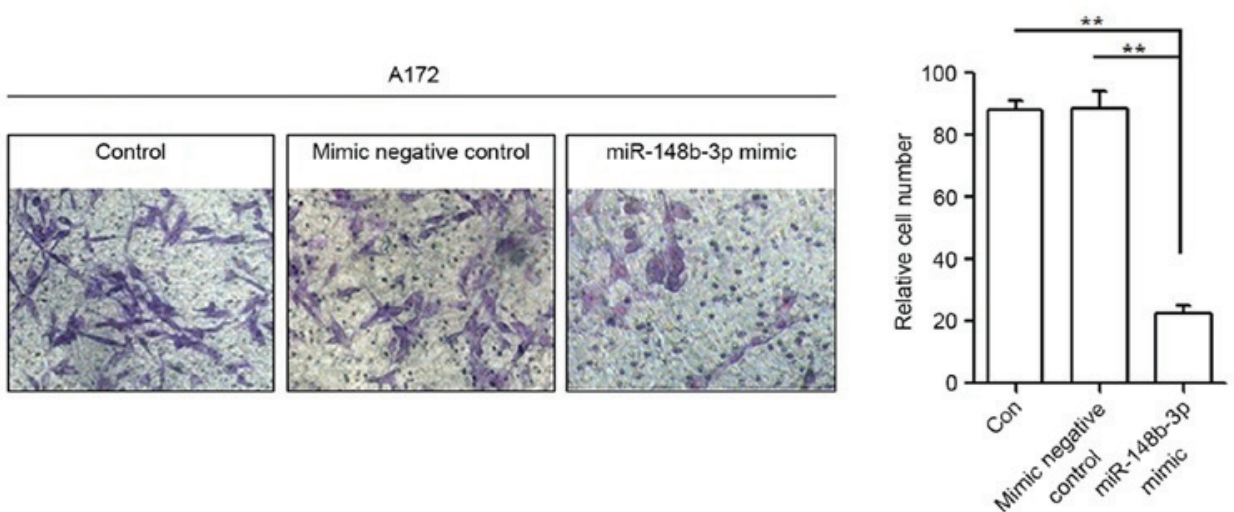

Figure 4. miR-148b-3p inhibits HOTAIR function. (A) Effect of miR-148b-3p mimic on cell proliferation in A172 cells, as determined using an MTT assay. ${ }^{*} \mathrm{P}<0.05,{ }^{* *} \mathrm{P}<0.01$ vs. mimic negative control group. (B) Effect of miR-148b-3p mimic on the cell cycle in A172 cells, evaluated by flow cytometry analysis. (C) Microscopic images (Giemsa staining; magnification, $x 400$ ) and quantification showing the effect of miR-148b-3p mimic on A172 cell invasive, evaluated by a Matrigel invasion assay. ${ }^{* *} \mathrm{P}<0.01$. Data are presented as the mean \pm standard deviation of three independent experiments. miR, microRNA.

Transient transfection with the miR-148b-3p mimic decreased the proliferation of A172 cells by $\sim 15 \%$ at $72 \mathrm{~h}$ compared with the mimic negative control group ( $\mathrm{P}=0.002$; Fig. 4A). The miR-148b-3p mimic also increased the cell population in the G1 phase and decreased the S-phase population (Fig. 4B), demonstrating that miR-148b-3p blocks the cell cycle of A172 cells. Furthermore, the Transwell invasion assay results clearly revealed that the miR-148b-3p mimic decreased cell invasion to $\sim 70 \%$ compared with the untreated and miR-148b-3p mimic negative controls ( $\mathrm{P}=0.002$; Fig. 4C).

\section{Discussion}

Years of research have revealed the essential role of non-coding lncRNAs and miRNAs in gene regulation, and their involvement in diverse biological processes, such as development and disease. Recently, rapid advances in bioinformatic analysis have expanded understanding of the transcriptome to a genome-wide level, and the interaction between lncRNA and miRNA has been shown to provide an additional element of control in gene regulation (43-45). In support an interaction 
between miRNA and lncRNA, the present study demonstrated the reciprocal repression of miRNA-148b and HOTAIR. The addition of a miR-148b-3p mimic decreased the expression levels of HOTAIR, while miR-148b-3p inhibitor increased the levels of HOTAIR. In addition, knockdown of HOTAIR induced the upregulation of miR-148b-3p.

HOTAIR is a 2,158-bp lncRNA that is located within the HOXC cluster of chromosome 12, and is flanked by $\mathrm{HOXC11}$ and HOXC12. HOTAIR activity is partially regulated by its interaction with PRC2, which is comprised of EZH2 (a histone H3K27 methylase), SUZ12 and EED. Since Gupta et al (41) first observed that loss of HOTAIR can inhibit the invasiveness of cancer cells, increasing evidence for a significant role of HOTAIR in carcinogenesis has been documented for several types of cancer, including glioma. The current study confirmed that HOTAIR is highly expressed in glioma tissues and the A172 glioma cell line. Furthermore, knockdown of HOTAIR decreased growth rates, blocked the cell cycle and suppressed the invasion of glioma cells, as well as increasing the expression of miR-148b-3p.

Previous studies have shown that miR-148b-3p is dysregulated in numerous types of cancer (46-48). For example, in colorectal cancer, it is downregulated and acts as a tumor suppressor by targeting the $C C K B R$ gene (38). miR-148b-3p is also downregulated in pancreatic cancer and suppresses cell growth by targeting the AMPK 1 gene (49). However, it is upregulated in ovarian cancer and may be involved in the early stages of ovarian carcinogenesis (50). These results reflect the controversial roles of miR-148b-3p in different types of cancer. To the best of our knowledge, the present study is the first to demonstrate that miR-148b-3p is downregulated in glioma cells. Additionally, an association between miR-148b-3p and lncRNA expression in cancer has not been reported until now. In the current study, luciferase assays indicated that miR-148b-3p reduces HOTAIR expression through the putative miR-148b-3p binding site in HOTAIR. To investigate whether miR-148b-3p has a metastasis-suppressing function in glioma cells, a miR-148b-3p mimic was transfected into A172 cells, resulting in reduced expression levels of HOTAIR. The data also showed that miR-148b-3p inhibits cell proliferation, blocks the cell cycle and reduces cell invasion.

In conclusion, the results of the current study indicate that miR-148b-3p has a tumor-suppressive role by downregulating HOTAIR in glioma, providing evidence for the importance of the interaction between lncRNA and miRNA in gliomagenesis. These results will aid in providing novel considerations for the molecule-targeted therapies of glioblastoma. Additional investigations are underway to further investigate other molecules involved in the HOTAIR-miR-148b-3p interaction.

\section{Acknowledgements}

The current study was supported in part by research grants from the Natural Science Foundation of Zhejiang Province, Youth Fund Project (grant no. LQ12C07001), the Research Fund for the Doctoral Program of Higher Education of China (grant no. 20133322120002), Zhejiang Medical and Health Science and Technology Project (grant no. 2014KYA150), the Science and Technology Creative Activity Plan for University Students in Zhejiang Province (grant no. 2015R410050) and the National Training Programs of Innovation and Entrepreneurship for Undergraduates (grant no. 201410344004).

\section{References}

1. Mattick JS: Challenging the dogma: The hidden layer of non-protein-coding RNAs in complex organisms. BioEssays 25: 930-939, 2003

2. Mattick JS: RNA regulation: A new genetics? Nat Rev Genet 5: 316-323, 2004.

3. Szymanski M, Barciszewska MZ, Erdmann VA and Barciszewski J: A new frontier for molecular medicine: Noncoding RNAs. Biochim Biophys Acta 1756: 65-75, 2005.

4. Prasanth KV and Spector DL: Eukaryotic regulatory RNAs: An answer to the 'genome complexity' conundrum. Genes Dev 21: 11-42, 2007.

5. Amaral PP, Dinger ME, Mercer TR and Mattick JS: The eukaryotic genome as an RNA machine. Science 319: 1787-1789, 2008.

6. Mercer TR, Dinger ME and Mattick JS: Long non-coding RNAs: Insights into functions. Nat Rev Genet 10: 155-159, 2009.

7. Geisler S and Coller J: RNA in unexpected places: Long non-coding RNA functions in diverse cellular contexts. Nat Rev Mol Cell Biol 14: 699-712, 2013.

8. Wilusz JE, Sunwoo H and Spector DL: Long noncoding RNAs: Functional surprises from the RNA world. Genes Dev 23: 1494-1504, 2009

9. Nagano T and Fraser P: No-nonsense functions for long noncoding RNAs. Cell 145: 178-181, 2011.

10. Zhang XQ and Leung GK: Long non-coding RNAs in glioma: Functional roles and clinical perspectives. Neurochem Int 77: 78-85, 2014.

11. Mercer TR and Mattick JS: Structure and function of long noncoding RNAs in epigenetic regulation. Nat Struct Mol Biol 20: 300-307, 2013.

12. Qureshi IA, Mattick JS and Mehler MF: Long non-coding RNAs in nervous system function and disease. Brain Res 1338: 20-35, 2010.

13. Mercer TR, Dinger ME, Sunkin SM, Mehler MF and Mattick JS: Specific expression of long noncoding RNAs in the mouse brain. Proc Natl Acad Sci USA 105: 716-721, 2008.

14. Ravasi T, Suzuki H, Pang KC, Katayama S, Furuno M, Okunishi R, Fukuda S, Ru K, Frith MC, Gongora MM, et al: Experimental validation of the regulated expression of large numbers of non-coding RNAs from the mouse genome. Genome Res 16: 11-19, 2006.

15. Carninci P, Kasukawa T, Katayama S, Gough J, Frith MC, Maeda N, Oyama R, Ravasi T, Lenhard B, Wells C, et al; RIKEN Genome Exploration Research Group and Genome Science Group (Genome Network Project Core Group): The transcriptional landscape of the mammalian genome. Science 309: $1559-1563,2005$

16. Kiang KM, Zhang XQ and Leung GK: Long Non-Coding RNAs: The Key Players in Glioma Pathogenesis. Cancers (Basel) 7: 1406-1424, 2015.

17. Louis DN, Ohgaki H, Wiestler OD, Cavenee WK, Burger PC, Jouvet A, Scheithauer BW and Kleihues P: The 2007 WHO classification of tumours of the central nervous system. Acta Neuropathol 114: 97-109, 2007.

18. Hatanpaa KJ, Burma S, Zhao D and Habib AA: Epidermal growth factor receptor in glioma: Signal transduction, neuropathology, imaging, and radioresistance. Neoplasia 12:675-684, 2010.

19. Yan H, Parsons DW, Jin G, McLendon R, Rasheed BA, Yuan W, Kos I, Batinic-Haberle I, Jones S, Riggins GJ, et al: IDH1 and IDH2 mutations in gliomas. New Eng N Engl J Med 360: 765-773, 2009.

20. Endersby R and Baker SJ: PTEN signaling in brain: Neuropathology and tumorigenesis. Oncogene 27: 5416-5430, 2008.

21. Gordon FE, Nutt CL, Cheunsuchon P, Nakayama Y, Provencher KA, Rice KA, Zhou Y, Zhang X and Klibanski A: Increased expression of angiogenic genes in the brains of mouse meg3-null embryos. Endocrinology 151: 2443-2452, 2010.

22. Shi Y, Wang Y, Luan W, Wang P, Tao T, Zhang J, Qian J, Liu N and You Y: Long non-coding RNA H19 promotes glioma cell invasion by deriving miR-675. PLoS One 9: e86295, 2014.

23. Wang P, Ren Z and Sun P: Overexpression of the long non-coding RNA MEG3 impairs in vitro glioma cell proliferation. J Cell Biochem 113: 1868-1874, 2012. 
24. Barsyte-Lovejoy D, Lau SK, Boutros PC, Khosravi F, Jurisica I, Andrulis IL, Tsao MS, Penn LZ: The c-Myc oncogene directly induces the H19 noncoding RNA by allele-specific binding to potentiate tumorigenesis. Cancer Res 66: 5330-5337, 2006.

25. Ellis BC, Molloy PL, Graham LD: CRNDE: A long non-coding RNA involved in CanceR, Neurobiology, and DEvelopment. Front Genet 3: 270, 2012.

26. Zhang X, Sun S, Pu JK, Tsang AC, O, Lee D, Man VO, Lui WM, Wong ST and Leung GK: Long non-coding RNA expression profiles predict clinical phenotypes in glioma. Neurobiol Dis 48: 1-8, 2012.

27. Zhou Y, Zhong Y, Wang Y, Zhang X, Batista DL, Gejman R, Ansell PJ, Zhao J, Weng C and Klibanski A: Activation of p53 by MEG3 non-coding RNA. J Biol Chem 282: 24731-24742, 2007.

28. Zhang K, Sun X, Zhou X, Han L, Chen L, Shi Z, Zhang A, Ye M, Wang Q, Liu C, et al: Long non-coding RNA HOTAIR promotes glioblastoma cell cycle progression in an EZH2 dependent manner. Oncotarget 6: 537-546, 2015.

29. Ma MZ, Li CX, Zhang Y, Weng MZ, Zhang MD, Qin YY, Gong W and Quan ZW: Long non-coding RNA HOTAIR, a c-Myc activated driver of malignancy, negatively regulates miRNA-130a in gallbladder cancer. Mol Cancer 13: 156, 2014.

30. Liu Q, Huang J, Zhou N, Zhang Z, Zhang A, Lu Z, Wu F and Mo YY: LncRNAloc285194 is a p53-regulated tumor suppressor. Nuclei Acids Res 41: 4976 4987, 2013

31. Zhang Z, Zhu Z, Watabe K, Zhang X, Bai C, Xu M, Wu F and Mo YY: Negative regulation of lncRNA GAS5 by miR-21. Cell Death Differ 20: 1558-1568, 2013.

32. Jalali S, Bhartiya D, Lalwani MK, Sivasubbu S and Scaria V: Systematic transcriptome wide analysis of IncRNA-miRNA interactions. PLoS One 8: e53823, 2013.

33. Juan L, Wang G, Radovich M, Schneider BP, Clare SE, Wang Y and Liu Y: Potential roles of microRNAs in regulating long intergenic noncodingRNAs. BMC Med Genomics 6 (Suppl 1): S7, 2013.

34. Braconi C, Kogure T, Valeri N, Huang N, Nuovo G, Costinean S, Negrini M, Miotto E, Croce CM and Patel T: microRNA-29 can regulate expression of the long non-coding RNA gene MEG3 in hepatocellular cancer. Oncogene 30: 4750-4756, 2011.

35. Tuo YL, Li XM and Luo J: Long noncoding RNA UCA1 modulates breast cancer cell growth and apoptosis through decreasing tumor suppressive miR-143. Eur Rev Med Pharmacol Sci 19: 3403-3411, 2015.

36. Song YX, Yue ZY, Wang ZN, Xu YY, Luo Y, Xu HM, Zhang X, Jiang L, Xing CZ and Zhang Y: MicroRNA-148b is frequently down-regulated in gastric cancer and acts as a tumor suppressor by inhibiting cell proliferation. Mol Cancer 10: 1, 2011.

37. Zhao Y, Jia HL, Zhou HJ, Dong QZ, Fu LY, Yan ZW, Sun J, Ren N, $\mathrm{Ye} \mathrm{QH}$ and Qin LX: Identification of metastasis-related microRNAs of hepatocellular carcinoma in hepatocellular carcinoma cell lines by quantitative real time PCR. Zhonghua Gan Zang Bing Za Zhi 17: 526-530, 2009 (In Chinese).
38. Song Y, Xu Y, Wang Z, Chen Y, Yue Z, Gao P, Xing C and $\mathrm{Xu}$ H: MicroRNA-148b suppresses cell growth by targeting cholecystokinin-2 receptor in colorectal cancer. Int J Cancer 131: 1042-1051, 2012.

39. Zhang JG, Shi Y, Hong DF, Song M, Huang D, Wang CY and Zhao G: MiR-148b suppresses cell proliferation and invasion in hepatocellular carcinoma by targeting WNT $1 / \beta$-catenin pathway. Sci Rep 5: 8087, 2015.

40. Livak KJ and Schmittgen TD: Analysis of relative gene expression data using real-time quantitative PCR and the 2(-Delta Delta C(T)) Method. Methods 25: 402-408, 2001

41. Gupta RA, Shah N, Wang KC, Kim J, Horlings HM, Wong DJ, Tsai MC, Hung T, Argani P, Rinn JL, et al: Long non-coding RNA HOTAIR reprograms chromatin state to promote cancer metastasis. Nature 464: 1071-1076, 2010.

42. Li JH, Liu S, Zhou H, Qu LH and Yang JH: starBase v2.0: Decoding miRNAceRNA, miRNA-ncRNA and protein-RNA interaction networks from largescaleCLIP-Seq data. Nucleic Acids Res 42: D92-D97, 2014

43. Guo L, Zhao Y, Yang S, Zhang H and Chen F: An integrated analysis of miRNA, lncRNA, and mRNA expression profiles. Biomed Res Int 2014: 345605, 2014.

44. Wu Q, Guo L, Jiang F, Li L, Li Z and Chen F: Analysis of the miRNA-mRNA-lncRNA networks in ER+ and ER- breast cancer cell lines. J Cell Mol Med 19: 2874-2887, 2015.

45. Yoon JH, Abdelmohsen K and Gorospe M: Functional interactions among microRNAs and long noncoding RNAs. Semin Cell Dev Biol 34: 9-14, 2014.

46. Zhou Z, Su Y and Fa X: Restoration of BRG1 inhibits proliferation and metastasis of lung cancer by regulating tumor suppressor miR-148b. Onco Targets Ther 8: 3603-3612, 2015.

47. Ghasemkhani N, Shadvar S, Masoudi Y, Talaei AJ, Yahaghi E, Goudarzi PK and Shakiba E. Down-regulated microRNA 148b expression as predictive biomarker and its prognostic significance associated with clinicopathological features in non-small-cell lung cancer patients. Diagn Pathol 10: 164, 2015.

48. Cimino D, De Pitta C,BearF, Casara S,Zampini M, RomualdiC, Damascus C, Pinatel E, Ponzone R, Brisken C, et al: miR-148b is a major coordinator in a relapse-associated miR signature in breast tumors. FEBS J 278: 199-200, 2011.

49. Zhao G, Zhang JG, Liu Y, Qin Q, Wang B, Tian K, Liu L, Li X, Niu Y, Deng SC, et al: miR-148b functions as a tumor suppressor in pancreatic cancer by targeting AMPK $\alpha 1$. Mol Cancer Ther 12: 83-93, 2013.

50. Chang H, Zhou X, Wang ZN, Song YX, Zhao F, Gao P, Chiang $\mathrm{Y}$ and $\mathrm{Xu} \mathrm{HM}$ : Increased expression of miR-148b in ovarian carcinoma and its clinical significance. Mol Med Rep 5: 1277-1280, 2012. 